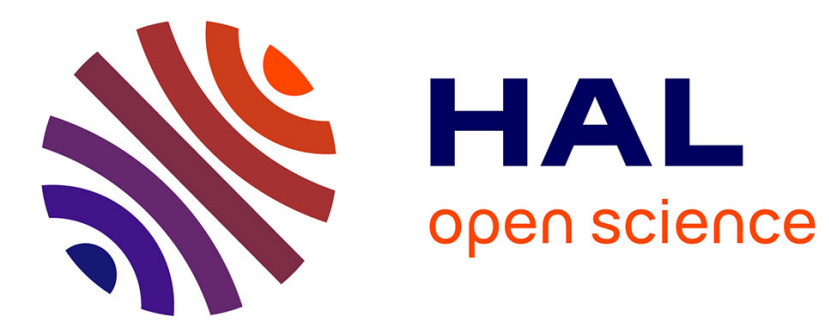

\title{
On the ideal triangulation graph of a punctured surface
}

\author{
Mustafa Korkmaz, Athanase Papadopoulos
}

\section{To cite this version:}

Mustafa Korkmaz, Athanase Papadopoulos. On the ideal triangulation graph of a punctured surface. Annales de l'Institut Fourier, 2012, 62 (4), p. 1367-1382. 10.5802/aif.2725 . hal-00423547

\section{HAL Id: hal-00423547 https://hal.science/hal-00423547}

Submitted on 12 Oct 2009

HAL is a multi-disciplinary open access archive for the deposit and dissemination of scientific research documents, whether they are published or not. The documents may come from teaching and research institutions in France or abroad, or from public or private research centers.
L'archive ouverte pluridisciplinaire HAL, est destinée au dépôt et à la diffusion de documents scientifiques de niveau recherche, publiés ou non, émanant des établissements d'enseignement et de recherche français ou étrangers, des laboratoires publics ou privés. 


\title{
ON THE IDEAL TRIANGULATION GRAPH OF A PUNCTURED SURFACE
}

\author{
MUSTAFA KORKMAZ AND ATHANASE PAPADOPOULOS
}

\begin{abstract}
We study the ideal triangulation graph $T(S)$ of a punctured surface $S$ of finite type. We show that if $S$ is not the sphere with at most three punctures or the torus with one puncture, then the natural map from the extended mapping class group of $S$ into the simplicial automorphism group of $T(S)$ is an isomorphism. We also show that under the same conditions on $S$, the graph $T(S)$ equipped with its natural simplicial metric is not Gromov hyperbolic. Thus, from the point of view of Gromov hyperbolicity, the situation of $T(S)$ is different from that of the curve complex of $S$.
\end{abstract}

AMS Mathematics Subject Classification: Primary 32G15. Secondary 20F38; 30F10.

Keywords: mapping class group ; surface ; arc complex ; ideal triangulation graph ; curve complex ; Gromov hyperbolic.

\section{INTRODUCTION}

In this paper, $S$ is a connected orientable surface of finite type, of genus $g \geq 0$ without boundary and with $n \geq 1$ punctures. We shall assume that the Euler characteristic $\chi(S)$ of $S$ is negative. The mapping class group of $S$, denoted by $\operatorname{Mod}(S)$, is the group of isotopy classes of orientation-preserving homeomorphisms of $S$. The extended mapping class group of $S, \operatorname{Mod}^{*}(S)$, is the group of isotopy classes of all homeomorphisms of $S$.

We denote by $\bar{S}$ the surface obtained from $S$ by filling in the punctures. Thus, $\bar{S}$ is a closed surface of genus $g$. The punctures can also be considered as distinguished points on $\bar{S}$, and we denote by $B \subset \bar{S}$ this set of distinguished points. An arc in $S$ (or in $\bar{S}$ ) is the image in $\bar{S}$ of a closed interval whose interior is homeomorphically embedded in $\bar{S} \backslash B$ and whose endpoints are on $B$. An arc in $S$ (or in $\bar{S}$ ) is said to be essential if it is not homotopic (relative to $B$ ) to a point in $\bar{S}$.

All homotopies of arcs considered in this paper are relative to their endpoints. An ideal triangulation (or, for short, a triangulation) of $S$ is a maximal collection of disjoint essential arcs that are pairwise non-homotopic. An essential arc that belongs to a triangulation will also be called an edge of that triangulation. A triangulation will sometimes be identified with the union of its edges, and will therefore be considered as a subset of $S$. A face of a triangulation $\Delta$ is a connected component of the complement of $\Delta$ in $S$ (or the closure of such a component), and it will also be called a triangle.

An elementary move is the operation of obtaining a triangulation from a given one by removing an edge and replacing it by a distinct edge. If $a$

Date: October 12, 2009. 
is the edge that is removed, then we shall say that the elementary move is performed on $a$.

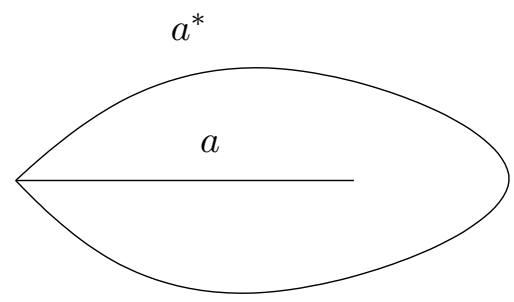

FiguRE 1. The edge $a$ is a non-exchangeable arc in a triangulation, and $a^{*}$ is its dual edge.

An edge $a$ in a triangulation $\Delta$ is said to be exchangeable if one can perform on $\Delta$ an elementary move, obtaining a new triangulation in which $a$ is replaced by an edge distinct from $a$. Otherwise, the edge is said to be non-exchangeable. It follows from the classification of surfaces that an edge in a triangulation $\Delta$ is non-exchangeable if and only if it joins two distinct punctures and if this edge is in the closure of a unique face of $\Delta$. In this case, there is a well-defined edge $a^{*}$ in $\Delta$ which is associated to $a$, which we call the edge dual to $a$, and which is the third edge of the unique triangle of $\Delta$ to which $a$ belongs. This situation is described in Figure 1 .

In this paper, we study the ideal triangulation graph of $S$. This is the simplicial graph $T(S)$ whose vertices are isotopy classes of triangulations of $S$ in which an edge connects two vertices whenever these two vertices differ by an elementary move. The extended mapping class group $\operatorname{Mod}^{*}(S)$ acts naturally on $T(S)$ by simplicial automorphisms. We prove the following.

Theorem 1.1. Let $S$ be a connected orientable surface with at least one puncture. If $S$ is not a sphere with at most three punctures or a torus with one puncture, then the natural homomorphism $\operatorname{Mod}^{*}(S) \rightarrow \operatorname{Aut}(T(S)$ is an isomorphism.

Note that the hypothesis on $S$ made in this theorem, combined with the condition $\chi(S) \leq-1$, is equivalent to $\chi(S) \leq-2$.

The proof of Theorem 1.1 involves the consideration of the arc complex of $S$. This is the abstract simplicial complex, denoted by $A(S)$, whose $k$ simplices, for each $k \geq 0$, are the collections of $k+1$ distinct isotopy classes of essential arcs on $S$ which can be represented by pairwise disjoint arcs on this surface. The ideal triangulation graph has been studied in [2] and [3]. The arc complex has been studied in [6], [4] and [5]. The idea of the proof of Theorem 1.1 follows a general scheme that was used in [4] and [9].

In the proof of Theorem 1.1, we shall use the following analogue of that theorem for the arc complex, which was obtained by Irmak and McCarthy:

Theorem 1.2. (河) Let $S$ be a connected orientable surface with at least one puncture. If the surface $S$ is not a sphere with at most three punctures or a torus with one puncture, then the natural homomorphism $\operatorname{Mod}^{*}(S) \rightarrow$ $\operatorname{Aut}(A(S))$ is an isomorphism. 
The graph $T(S)$ is the one-skeleton of the simplicial complex dual to the arc complex $A(S)$. Thus, any automorphism of $A(S)$ induces an automorphism of the graph $T(S)$. But since $T(S)$ is a strict subcomplex of the dual complex of $A(S)$, its automorphism group could a priori be larger than the automorphism group of $A(S)$. Theorem 1.1 shows that this is not the case.

There are three special cases of surfaces that admit ideal triangulations and that are excluded by the hypothesis of Theorem 1.1: the cases of a sphere with two or three punctures and the case of a torus with one puncture. Let us briefly discuss these cases.

If $S$ is a sphere with two punctures, then $T(S)$ consists of only one vertex, hence its automorphism group is trivial. But the extended mapping class group $\operatorname{Mod}^{*}(S)$ is isomorphic to Klein's four group, the direct sum of two cyclic groups of order two. Thus, the natural homomorphism $\operatorname{Mod}^{*}(S) \rightarrow$ $\operatorname{Aut}(T(S))$ is surjective and not injective.

If $S$ is a sphere with three punctures, then any ideal triangulation of $S$ has three vertices, three edges and two faces. The simplicial complex $T(S)$ is a finite graph, homeomorphic to a tripod. The center of the tripod corresponds to the ideal triangulation of $S$ in which every edge is exchangeable, and the three other vertices of $T(S)$ correspond to ideal triangulations in which exactly one edge is exchangeable. The automorphism group Aut $(T(S))$ of $T(S)$ is isomorphic to the permutation group on three elements (the three vertices of valency one of the tripod). The mapping class group of $S$ is also isomorphic to the permutation group on three element (the three punctures of $S$ ), and the natural homomorphism $\operatorname{Mod}(S) \rightarrow \operatorname{Aut}(T(S))$ is an isomorphism. The natural homomorphism $\operatorname{Mod}^{*}(S) \rightarrow \operatorname{Aut}(T(S))$ is surjective and its kernel is the center of $\operatorname{Mod}^{*}(S)$, which is a cyclic group of order two.

If $S$ is a torus with one puncture, then the ideal triangulation graph $T(S)$ is a regular infinite tree in which every vertex has valency 3 . The automorphism group of such a tree is uncountable. To see this, we consider the set of one-sided infinite sequences of letters on the alphabet $\{f, n\}$. This set of sequences is uncountable, and it can be injected in the simplicial automorphism group of the tree. Such an injection can be done by choosing a base point of the tree, then embedding the set of one-sides sequences as the set of simplicial geodesic rays starting at that base point, and finally defining for each such geodesic ray an automorphisms of the tree by interpreting the letter $f$ as a flip, and the letter $n$ as no flip. Thus, in the case considered, the natural homomorphism $\operatorname{Mod}^{*}(S) \rightarrow \operatorname{Aut}(T(S))$ is highly non-surjective since the extended mapping class group $\operatorname{Mod}^{*}(S)$ is countable.

By declaring that the length of each edge is one, we may consider $T(S)$ as a metric space. With this metric, the length of a simplicial path between two vertices is the number of edges in that path. It is now natural to ask whether $T(S)$ is Gromov-hyperbolic.

Theorem 1.1 implies the following:

Theorem 1.3. Let $S$ be a connected orientable surface with at least one puncture. Suppose that $S$ is not a sphere with at most three punctures or a torus with one puncture. Then the ideal triangulation graph of $S$ is not Gromov-hyperbolic. 
Proof. The action of the extended mapping class group $\operatorname{Mod}^{*}(S)$ on the vertices of $T(S)$ is free, since a homeomorphism of $S$ which fixes the homotopy class of an ideal triangulation is homotopic to the identity. The action of $\operatorname{Mod}^{*}(S)$ on $T(S)$ is co-compact, since up to homeomorphisms, there are only finitely homotopy classes of ideal triangulations on any surface of finite type. This is because the surface $S$ equipped with any ideal triangulation is obtained by gluing $4 g-4+2 n$ triangles along edges, and there are only finitely many ways to glue such a finite set of triangles to get a triangulated surface. Thus, if $T(S)$ were Gromov-hyperbolic, then $\operatorname{Mod}^{*}(S)$ would be word-hyperbolic (see [1]). But under the hypotheses of the theorem, the extended mapping class group $\operatorname{Mod}^{*}(S)$ is not word-hyperbolic, since it contains free abelian groups of rank $\geq 2$.

We note that in the cases excluded in the hypothesis of Corollary 1.3, the situations are as follows:

- If $S$ is a sphere with one puncture, then the triangulation graph $T(S)$ is empty.

- If $S$ is a sphere with two punctures, then $T(S)$ consists of only one vertex, hence it is hyperbolic.

- If $S$ is a sphere with three punctures, then its triangulation graph is compact, and therefore hyperbolic.

- If $S$ is a torus with one puncture, then its triangulation graph is hyperbolic since, as we recalled above, it is a tree.

Theorem 1.3 says that the large-scale geometry of the triangulation graph of $S$ is different from that of the curve complex, which, by a result of Masur and Minsky, is hyperbolic.

\section{Squares and pentagons in $T(S)$}

To simplify notation, we shall often identify an arc or a triangulation on $S$ with its isotopy class.

We shall use two special classes of simplicial closed paths in $T(S)$, and we now describe them.

A square in $T(S)$ is a simple closed (that is, injective) path in this graph consisting of four edges, as represented in Figure 2. In this figure, elementary moves are performed on exchangeable edges $a$ and $c$, whose images under these moves are denoted, respectively, by $a^{\prime}$ and $c^{\prime}$. Note that the existence of such an elementary move implies that the interiors of the faces containing $a$ and $c$ in the surface $S$ are disjoint. The move is also represented diagrammatically as follows:

$$
\langle a, c\rangle \leftrightarrow\left\langle a, c^{\prime}\right\rangle \leftrightarrow\left\langle a^{\prime}, c^{\prime}\right\rangle \leftrightarrow\left\langle a^{\prime}, c\right\rangle \leftrightarrow\langle a, c\rangle .
$$

In this notation, a symbol such as $\langle a, c\rangle \leftrightarrow\left\langle a, c^{\prime}\right\rangle$ represents an elementary move between two triangulations, the first one having $a$ and $c$ among its edges and the second one having $a$ and $c^{\prime}$ among its edges, and where the move is performed on $c$, which is transformed into $c^{\prime}$. All other edges remain unchanged.

A pentagon in $T(S)$ is a simple closed path in $T(S)$ of length five, represented diagrammatically in Figure 3. In that figure, at each vertex, we have 
indicated the name of a pair of edges, one which remains invariant after a move represented by an edge adjacent to that vertex, and one which is transformed by that move into an edge whose name appears in the label of the corresponding adjacent vertex. This move is also represented diagrammatically as follows:

$$
\langle a, b\rangle \leftrightarrow\langle a, e\rangle \leftrightarrow\langle e, d\rangle \leftrightarrow\langle d, c\rangle \leftrightarrow\langle c, b\rangle \leftrightarrow\langle b, a\rangle .
$$

Lemma 2.1. There are no closed paths of length three in $T(S)$.

Proof. Assume there exists such a path and let us represent it by a diagram

$$
\langle a, b\rangle \leftrightarrow\langle b, c\rangle \leftrightarrow\langle c, a\rangle \leftrightarrow\langle a, b\rangle
$$

in which $a$ and $c$ are distinct arcs and where the double arrows represent, as before, elementary moves, that is, the $\operatorname{arc} a$ is transformed into the $\operatorname{arc} c$ by the first move, and so on. But the existence of the moves $\langle a, b\rangle \leftrightarrow\langle b, c\rangle$ and $\langle a, b\rangle \leftrightarrow\langle c, a\rangle$ imply $a=b$, a contradiction. This proves the lemma.

Lemma 2.2. Every simple closed path of length four in $T(S)$ is a square (that is, it is of the form described in Figure Q2).

Proof. Consider a simple closed path of length four in $T(S)$, and let us label it as

$$
\langle a, b\rangle \leftrightarrow\langle a, c\rangle \leftrightarrow\langle c, d\rangle \leftrightarrow\langle d, e\rangle \leftrightarrow\langle a, b\rangle .
$$

Then, the existence of the last move implies that either $d=a$, or $d=b$, or $e=a$, or $e=b$. We analyze each case separately.

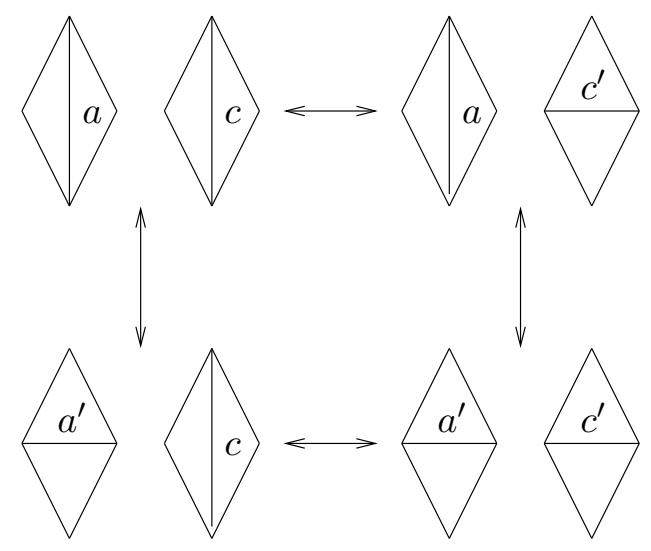

FiguRE 2. A square in the triangulation graph.

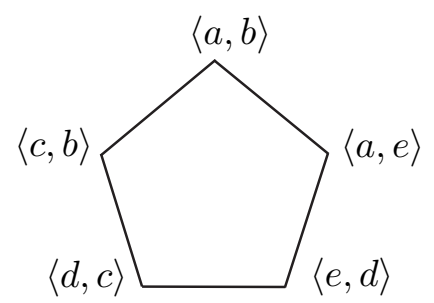

FigURE 3. A pentagon in the triangulation graph. 
- The case $d=a$ is excluded, because of the existence of the move $\langle a, c\rangle \leftrightarrow$ $\langle c, d\rangle$.

- If $d=b$ then we get two moves $\langle b, e\rangle \leftrightarrow\langle a, b\rangle$ and $\langle c, b\rangle \leftrightarrow\langle b, e\rangle$ which give $a=c$, which is excluded since we have a vertex labelled $\langle a, c\rangle$ in the path.

- If $e=a$ then we get two moves $\langle a, b\rangle \leftrightarrow\langle a, c\rangle$ and $\langle a, d\rangle \leftrightarrow\langle a, b\rangle$ which gives $d=c$, which is excluded since we have a vertex labelled $\langle c, d\rangle$ in the path.

- The remaining case is $e=b$, which labels the simple closed path in the following way:

$$
\langle a, b\rangle \leftrightarrow\langle a, c\rangle \leftrightarrow\langle c, d\rangle \leftrightarrow\langle d, b\rangle \leftrightarrow\langle a, b\rangle,
$$

which is of the form represented in Figure 2.

Lemma 2.3. Every simple closed path of length five in $T(S)$ is a pentagon (that is, it is of the form described in Figure 3).

Proof. The proof is by inspection, like the proof of Lemma 2.2 (and it uses Lemma 2.1).

In the next two lemmas, and later in the paper, if $\Delta$ and $\Delta^{\prime}$ are two triangulations of $S$ connected by an edge in $T(S)$, then the notation $\Delta-\Delta^{\prime}$ will be used to denote (the isotopy classes of) the edge in $\Delta$ that is not in $\Delta^{\prime}$.

Lemma 2.4. Consider a square in $T(S)$, represented as $\Delta_{1} \leftrightarrow \Delta_{2} \leftrightarrow \Delta_{3} \leftrightarrow$ $\Delta_{4} \leftrightarrow \Delta_{1}$. Then we have $\Delta_{1}-\Delta_{4}=\Delta_{2}-\Delta_{3}$.

Proof. This can be checked on Figure 2.

Lemma 2.5. Consider a pentagon in $T(S)$, represented as $\Delta_{1} \leftrightarrow \Delta_{2} \leftrightarrow$ $\Delta_{3} \leftrightarrow \Delta_{4} \leftrightarrow \Delta_{5} \leftrightarrow \Delta_{1}$. Then we have $\Delta_{1}-\Delta_{5}=\Delta_{2}-\Delta_{3}$.

Proof. This can be checked on Figure 3.

\section{Proof of Theorem 1.1}

Let $f: T(S) \rightarrow T(S)$ be a simplicial automorphism. We associate to $f$ a simplicial automorphism $\tilde{f}: A(S) \rightarrow A(S)$, defined as follows.

Let $a$ be an essential arc on $S$. We choose a triangulation $\Delta$ in which $a$ is an exchangeable edge. Such a triangulation always exists. Since $a$ is exchangeable in $\Delta$, we can perform an elementary move on $a$, replacing it by an $\operatorname{arc} a^{\prime}$. Let $\Delta_{a}$ be the triangulation obtained from $\Delta$ by this elementary move. Since $f$ is simplicial, the triangulations $f(\Delta)$ and $f\left(\Delta_{a}\right)$ (like $\Delta$ and $\Delta_{a}$ ) are joined by an edge in $T(S)$. In other words, the two triangulations $f(\Delta)$ and $f\left(\Delta_{a}\right)$ differ by an elementary move. We then define $\widetilde{f}(a)$ to be the edge that is in $f(\Delta)$ but not in $f\left(\Delta_{a}\right)$. In short, we have

$$
\widetilde{f}(a)=f(\Delta)-f\left(\Delta_{a}\right) .
$$

We now prove the following: 
Proposition 3.1. The map $\tilde{f}: A(S) \rightarrow A(S)$ is well-defined. That is, for any vertex $a$ in $A(S)$, the arc $\widetilde{f}(a)$ is independent of the choice of the triangulation $\Delta$ in which a is exhangeable.

Proof. Denoting a triangulation as the set of its edges, let $\Delta=\left\{a, c_{1}, \ldots, c_{k}\right\}$ and $\Delta^{\prime}=\left\{a, c_{1}^{\prime}, \ldots, c_{k}^{\prime}\right\}$ be two different triangulations used in the definition of $\widetilde{f}(a)$.

Let $R$ be the surface $S$ cut along $a$. There are two cases for the surface $R$ :

(1) $R$ has two boundary components coming from the curve $a$, and in this case there is one distinguished point on each of these boundary components, coming from the puncture at the endpoints of $a$. This occurs when the arc $a$ joins one puncture of $S$ to itself.

(2) $R$ has one boundary component, with two distinguished points on that boundary. This occurs when $a$ joins two distinct punctures on $S$.

The triangulations $\Delta$ and $\Delta^{\prime}$ naturally induce triangulations on $R$, with the labelling by edges, $\left\{a_{1}, a_{2}, c_{1}, \ldots, c_{k}\right\}$ and $\left\{a_{1}, a_{2}, c_{1}^{\prime}, \ldots, c_{k}^{\prime}\right\}$, where the edge $a$ has been replaced by two edges $a_{1}$ and $a_{2}$. In Case 11, each of the edges $a_{1}$ and $a_{2}$ appears on a boundary component of $R$, and in Case 2, the union of the edges $a_{1}$ and $a_{2}$ forms the boundary component of $R$, and there are two distinguished points on that boundary component.

In each case, we can join the two triangulations induced on $R$ by $\Delta$ and $\Delta^{\prime}$ by a finite sequence of elementary moves on $R$, in such a way that the two distinguished points and the two edges $a_{1}$ and $a_{2}$ on $R$ are left fixed by these elementary moves. This follows from Harer's result on the connectedness of the triangulation graph of a surface with boundary and with distinguished points on boundary components. (See [2]; the result is also cited in [3].)

Now gluing $a_{1}$ to $a_{2}$ back gives a simplicial path in $T(S)$ joining $\Delta$ to $\Delta^{\prime}$ such that each triangulation in this path contains $a$ as an edge. We denote the sequence of triangulations in this path by

$$
\Delta=\Delta_{0}, \Delta_{1}, \ldots, \Delta_{l}=\Delta^{\prime} .
$$

We can assume that this path is simple.

We shall use the following:

Lemma 3.2. We can assume that we can choose the path (11) in such a way that the edge $a$ is exchangeable in each triangulation representing a vertex of this path.

Proof. Suppose there is a vertex in the path (11) that represents a triangulation in which $a$ is not exchangeable, and let $\Delta_{i}$ be the triangulation represented by the first such vertex, after the vertex $\Delta$. Then, $\Delta_{i+1}$ (respectively $\Delta_{i-1}$ ) is the triangulation represented by the vertex after (respectively before) $\Delta_{i}$. Note that since $a$ is exchangeable in $\Delta$ and in $\Delta^{\prime}$, we have $1 \leq i<l$. There are two possibilities for the vertex joining $\Delta_{i}$ and $\Delta_{i+1}$, and they are represented respectively in Figure 1 (a) and (b).

The first possibility (top of Figure 1 (a)) is when the elementary move that takes $\Delta_{i}$ to $\Delta_{i+1}$ involves an edge that is on the boundary of a triangle 
having the edge $a^{*}$ (the dual of $a$ ) as an edge. (Note that we exclude the case where the elementary move is performed on the edge $a^{*}$, since in that case we recover the triangulation $\Delta_{i-1}$ as a result, but we assumed the path is simple.) In this case, we replace the subpath $\Delta_{i-1} \leftrightarrow \Delta_{i} \leftrightarrow \Delta_{i+1}$ by the path $\Delta_{i-1} \leftrightarrow \Delta_{i}^{\prime} \leftrightarrow \Delta_{i+1}^{\prime} \leftrightarrow \Delta_{i+1}$ represented in the bottom of Figure 4 (a).

The second possibility (top of Figure 1 (b)) is when the elementary move that takes $\Delta_{i}$ to $\Delta_{i+1}$ involves an edge that is not on the boundary of a triangle whose boundary contains $a^{*}$. In Figure (b), we have symbolically represented that elementary move on a quadrilateral that is disjoint from $a$ and $a^{*}$. In that case, we replace the subpath $\Delta_{i-1} \leftrightarrow \Delta_{i} \leftrightarrow \Delta_{i+1}$ by the path $\Delta_{i-1} \leftrightarrow \Delta_{i}^{\prime} \leftrightarrow \Delta_{i+1}$ represented in Figure 1 (b).

In each case, each vertex of the new simplicial path that joins $\Delta$ to $\Delta^{\prime}$ contains $a$ as an edge, and the number of occurrences of vertices in this path in which $a$ is non-exchangeable has been reduced by one. This allows us, by induction, to obtain a path joining $\Delta$ and $\Delta^{\prime}$ in $T(S)$, in which every vertex is represented by a triangulation that contains $a$ and where $a$ is exchangeable.

This proves Lemma 3.2

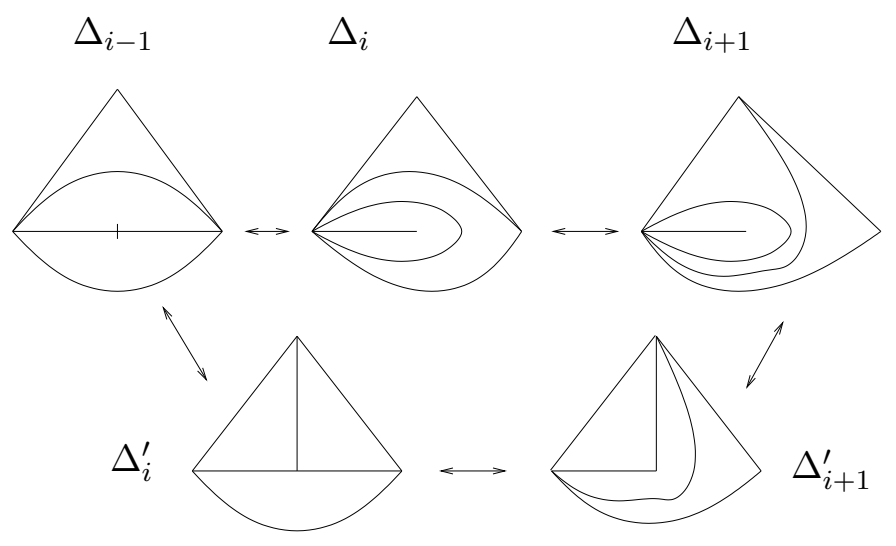

(a)

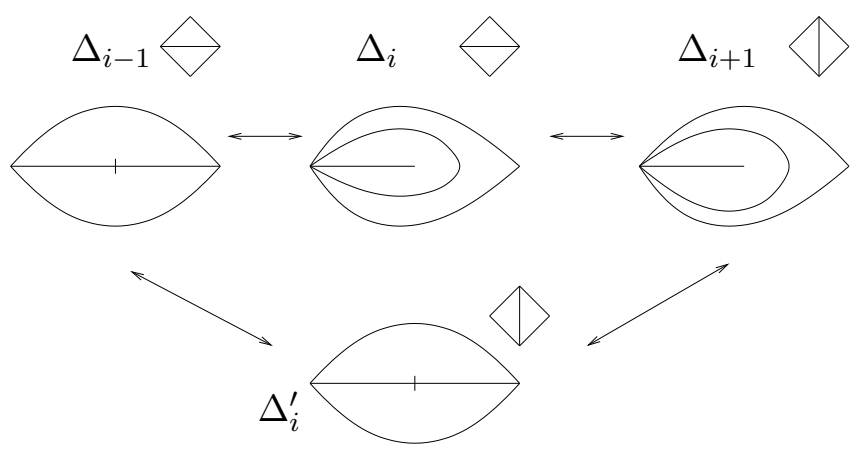

(b)

FigURE 4. Replacing a path in which each vertex contains $a$ by a path where at each vertex $a$ is an exchangeable edge. 
We now continue with the proof of Proposition 3.1.

Note that the closed paths in $T(S)$ that are represented in Figure 4 (a) and (b) are a pentagon and a square.

To prove that $\widetilde{f}$ is well-defined, by Lemma 3.2 it suffices to consider the case where $\Delta$ and $\Delta^{\prime}$ are joined by an edge in $T(S)$.

By reordering the edges of the triangulation $\Delta$, we can assume that $c_{1}$ is the vertex that is transformed by the elementary move that takes $\Delta$ to $\Delta^{\prime}$. We use as before the notation $\Delta \leftrightarrow \Delta^{\prime}$, with $\Delta=\left\langle a, c_{1}\right\rangle$ and $\Delta^{\prime}=\left\langle a, c_{1}^{\prime}\right\rangle$, to denote this elementary move, in which $c_{1}^{\prime}$ is the image of $c_{1}$. Edges other than $c_{1}$ are unchanged. We distinguish two cases:

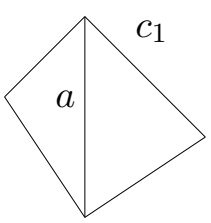

(a)

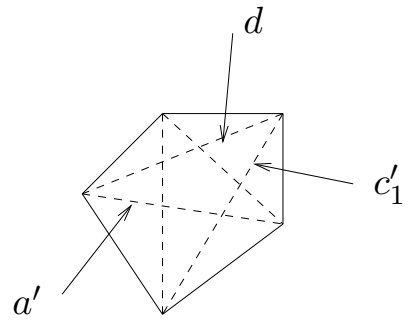

(b)

FiguRE 5. The star in the right hand side figure corresponds to a pentagon relation.

Case 1.- In the triangulation $\Delta, a$ and $c_{1}$ are two edges of a common triangle (see Figure 5 (a)). Then, since $S$ is not a torus with one puncture or a sphere with three punctures, the edge joining $\Delta$ and $\Delta^{\prime}$ in $T(S)$ belongs to a pentagon in this graph. This follows from the fact that both $a$ and $c_{1}$ are exchangeable, and it can be seen diagrammatically in Figure 5 (b), where the sequence of elementary moves representing the pentagon relation is:

$$
\left\langle a, c_{1}\right\rangle \leftrightarrow\left\langle a, c_{1}^{\prime}\right\rangle, \leftrightarrow\left\langle c_{1}^{\prime}, d\right\rangle, \leftrightarrow\left\langle d, a^{\prime}\right\rangle, \leftrightarrow\left\langle a^{\prime}, c_{1}\right\rangle, \leftrightarrow\left\langle c_{1}, a\right\rangle .
$$

Let $\mathcal{P}$ denote this pentagon. Since $f: T(S) \rightarrow T(S)$ is simplicial, the image $f(\mathcal{P})$ of $\mathcal{P}$ by $f$ is again a pentagon (Lemma 2.3). In the pentagon $\mathcal{P}$, the triangulation $\left\langle a, c_{1}\right\rangle$ is labelled $\Delta$, the triangulation $\left\langle a^{\prime}, c_{1}\right\rangle$ is labelled $\Delta_{a}$, the triangulation $\left\langle a, c_{1}^{\prime}\right\rangle$ is labelled $\Delta^{\prime}$ and the triangulation $\left\langle d, c_{1}^{\prime}\right\rangle$ is labelled $\Delta_{a}^{\prime}$. We then have, by Lemma 2.5 applied to the pentagon $f(\mathcal{P})$,

$$
f(\Delta)-f\left(\Delta_{a}\right)=f\left(\Delta^{\prime}\right)-f\left(\Delta_{a}^{\prime}\right) .
$$

This completes the proof of the fact the $\widetilde{f}(a)$ is well-defined in Case 1 .

Case 2.- In the triangulation $\Delta, a$ and $c_{1}$ are not edges of the same triangle. In this case, we can perform elementary moves on $a$ and $c_{1}$ independently from each other, and we obtain the following square in $T(S)$ :

$$
\left\langle a, c_{1}\right\rangle \leftrightarrow\left\langle a, c_{1}^{\prime}\right\rangle, \leftrightarrow\left\langle a_{1}^{\prime}, c_{1}^{\prime}\right\rangle, \leftrightarrow\left\langle a^{\prime}, c_{1}\right\rangle, \leftrightarrow\langle a, c\rangle .
$$

Similarly to the preceding case, the triangulation $\left\langle a, c_{1}\right\rangle$ is labelled $\Delta$, the triangulation $\left\langle a^{\prime}, c_{1}\right\rangle$ is labelled $\Delta_{a}$, the triangulation $\left\langle a, c_{1}^{\prime}\right\rangle$ is labelled $\Delta^{\prime}$ and the triangulation $\left\langle a_{1}^{\prime}, c_{1}^{\prime}\right\rangle$ is labelled $\Delta_{a}^{\prime}$, and we get again, this time using Lemma 2.4,

$$
f(\Delta)-f\left(\Delta_{0}\right)=f\left(\Delta^{\prime}\right)-f\left(\Delta_{0}^{\prime}\right)
$$


This shows that $\widetilde{f}(a)$ is well-defined in each case.

This completes the proof of Proposition 3.1.

The following naturality formula will be useful.

Proposition 3.3. For any ideal triangulation $\Delta=\left\{c_{0}, c_{1}, \ldots, c_{k}\right\}$, we have

$$
f(\Delta)=\left\{\widetilde{f}\left(c_{0}\right), \widetilde{f}\left(c_{1}\right), \ldots, \widetilde{f}\left(c_{k}\right)\right\} .
$$

Proof. It suffices to prove that $f(\Delta) \subset\left\{\widetilde{f}\left(c_{0}\right), \widetilde{f}\left(c_{1}\right), \ldots, \widetilde{f}\left(c_{k}\right)\right\}$. Let $a$ be any (homotopy class of) edge in $\Delta$. If $a$ is exchangeable, then it follows from the definition of $\widetilde{f}$ that $\widetilde{f}(a)$ is in the simplex $f(\Delta)$. Assume now that $a$ is not exchangeable, and let $a^{*}$ be its dual edge. Then $a^{*}$ is exchangeable in $\Delta$. Let $\Delta^{\prime}=\Delta_{a^{*}}$ be the ideal triangulation obtained from $\Delta$ by exchanging the edge $a^{*}$. Finally, let $\Delta_{a}^{\prime}$ be the ideal triangulation obtained from $\Delta^{\prime}$ by exchanging the edge $a$. Since there is an edge in $T(S)$ joining $\Delta$ to $\Delta^{\prime}$, and an edge in $T(S)$ joining $\Delta^{\prime}$ to $\Delta_{a}^{\prime}$, and since $f$ is simplicial, there is an edge in $T(S)$ joining $f(\Delta)$ to $f\left(\Delta^{\prime}\right)$, and an edge in $T(S)$ joining $f\left(\Delta^{\prime}\right)$ to $f\left(\Delta_{a}^{\prime}\right)$. By definition, $\widetilde{f}(a)$ belongs to the triangulation $f\left(\Delta^{\prime}\right)$. Then, the edge $\widetilde{f}(a)$ is transformed by the move $f\left(\Delta^{\prime}\right) \leftrightarrow f\left(\Delta_{a}^{\prime}\right)$. Therefore, $\widetilde{f}(a)$ is not an edge of $f\left(\Delta_{a}^{\prime}\right)$. But since $\widetilde{f}(a)$ is not transformed by the move $f(\Delta) \leftrightarrow f\left(\Delta^{\prime}\right)$, we conclude that $\widetilde{f}(a)$ is an edge of $f(\Delta)$.

Corollary 3.4. The map $\tilde{f}: A(S) \rightarrow A(S)$ is simplicial.

Proposition 3.5. If $f$ and $h$ are two automorphisms of $T(S)$, then $\widetilde{f h}=\widetilde{f h}$.

Proof. Let $a$ be (the isotopy class of) an arc on $S$. We show that $\widetilde{f h}(a)=$ $\widetilde{f}(\widetilde{h}(a))$.

Let $\Delta=\left\{a, c_{1}, c_{2}, \ldots, c_{k}\right\}$ be a triangulation in which $a$ is exchangeable and let $\Delta^{\prime}=\left\{a^{\prime}, c_{1}, c_{2}, \ldots, c_{k}\right\}$ be the triangulation obtained from $\Delta$ by an elementary move on $a$. Then, $\widetilde{h}(a)=h(\Delta)-h\left(\Delta^{\prime}\right)$, and $\widetilde{f h}(a)=f h(\Delta)-$ $f h\left(\Delta^{\prime}\right)$.

Since the map $h: T(S) \rightarrow T(S)$ is simplicial and since the triangulation $\Delta$ is related to $\Delta^{\prime}$ in $T(S)$ by an edge, the triangulation $h(\Delta)$ is also related to the triangulation $h\left(\Delta^{\prime}\right)$ by an edge. Since $h(\Delta)$ contains the $\operatorname{arc} \widetilde{h}(a)$ as an exchangeable arc, we can use $h(\Delta)$ and $h\left(\Delta^{\prime}\right)$ to define $\widetilde{f}(\widetilde{h}(a))$, and we obtain:

$$
\begin{aligned}
\widetilde{f}(\widetilde{h}(a)) & =f(h(\Delta))-f\left(h\left(\Delta^{\prime}\right)\right) \\
& =(f h)(\Delta)-(f h)\left(\Delta^{\prime}\right) \\
& =\widetilde{f h}(a) .
\end{aligned}
$$

This completes the proof.

Proposition 3.6. For any automorphism $f \in \operatorname{Aut}(T(S))$, the associated map $\tilde{f}: A(S) \rightarrow A(S)$ is an automorphism. 
Proof. Let $h=f^{-1} \in \operatorname{Aut}(T(S))$ be the inverse of $f$. By Proposition 3.5, we have $\widetilde{f} \widetilde{h}=\widetilde{\mathrm{I}}=\widetilde{h} \widetilde{f}$, where I is the identity map of $T(S)$. Now from the definitions, the map $\widetilde{\mathrm{I}}: A(S) \rightarrow A(S)$ associated to I is the identity map of $A(S)$. Thus, $\widetilde{f}$ has an inverse, which shows that $\widetilde{f}: A(S) \rightarrow A(S)$ is a bijection.

Since $\widetilde{f}$ is also simplicial by Corollary 3.4, it is an automorphism of $A(S)$.

Finally, we prove the following theorem which, together with Theorem 1.2, implies Theorem 1.1.

Theorem 3.7. Suppose the surface $S$ is not a sphere with at most three punctures or a torus with one puncture. Then the map $\phi: \operatorname{Aut}(T(S)) \rightarrow$ $\operatorname{Aut}(A(S))$ defined by $f \mapsto \widetilde{f}$ is an isomorphism.

Proof. Given the propositions that we already proved, it remains to show is that $\phi$ is bijective.

We first show that $\phi$ is onto. Let $h$ be an arbitrary element in $\operatorname{Aut}(A(S))$. By the result of Irmak McCarthy mentioned above (Theorem 1.2), $h$ is induced by a homeomorphism $H: S \rightarrow S$, which induces an automorphism $f$ of $T(S)$, and this automorphism satisfies $\widetilde{f}=h$. Thus, $\phi$ is onto.

Now we show that $f$ is one-to-one. Let $f$ be an element of $\operatorname{Aut}(T(S))$ such that $\widetilde{f}$ is the identity automorphism of $A(S)$. Then, by Proposition 3.3, $f$ acts trivially on $T(S)$. Thus, $f$ is one-to-one. This completes the proof of the theorem.

\section{REFERENCES}

[1] M. Gromov, Hyperbolic groups, In: Essays in Group Theory, edited by S.M. Gersten. MSRI Publications 8, Springer-Verlag, 1987, 75Đ263.

[2] J. L. Harer, Stability of the homology of the mapping class groups of orientable surfaces, Annals of Math. 121 (1985), 215-249.

[3] A. Hatcher, On triangulations of surfaces. Top. and its Appl. 40 (1991), 189-194. A new version is available in the author's webpage.

[4] E. Irmak, M. Korkmaz, Automorphisms of the Hatcher-Thurston complex. Isr. J. Math. 162, 183-196 (2007).

[5] E. Irmak and J. D. McCarthy, Injective simplicial maps of the arc complex, Turkish Journal of Mathematics, 33 (2009), 1-16.

[6] N. V. Ivanov, Automorphisms of Teichmüller modular groups. Lecture Notes in Math., No. 1346, Springer-Verlag, Berlin and New York, 1988, 199-270.

[7] M. Korkmaz, Automorphisms of complexes of curves on punctured spheres and on punctured tori. Topology and its Applications 95 no. 2; 85-111 (1999).

[8] F. Luo, Automorphisms of the complex of curves, Topology 39, no. 2, 283-298 (2000).

[9] D. Margalit, Automorphisms of the pants complex. Duke Math. J. 121, No. 3, 457-479 (2004).

Mustafa Korkmaz, Department of Mathematics, Middle East Technical University, 06531 AnkARA, Turkey.

E-mail address: korkmaz@metu.edu.tr

Athanase Papadopoulos, Max-Plank-Institut für Mathematik, Vivatsgasse 7, 53111 Bonn, Germany, and: Institut de Recherche Mathématique Avancée, Université de Strasbourg and CNRS, 7 rue René Descartes, 67084 Strasbourg Cedex, France.

E-mail address: papadopoulos@math.u-strasbg.fr 